\title{
Article
}

\author{
Doi 10.5943/sif/4/1/25
}

\section{Two new additions to mycoflora (Hyphomycetes) of India}

\author{
Verma RK ${ }^{1,2}$, Prasher $\mathrm{IB}^{2}$, Sushma ${ }^{2,3}$ and Gautam $\mathrm{AK}^{4}$ \\ ${ }^{1}$ Department of Botany, Dolphin PG College of science and Agriculture Chunni Kalan, Fatehgarh Sahib, Punjab, \\ 140307, India \\ ${ }^{2}$ Department of Botany, Mycology and Plant Pathology Laboratory, Panjab University Chandigarh, 160014, India \\ ${ }^{3}$ Department of Botany, Govt. PG College for Girls Sector- 42, Chandigarh, India \\ ${ }^{4}$ School of Agriculture, Faculty of Science, Abhilashi University, Mandi- Himachal Pradesh, 175028, India
}

Verma RK, Prasher IB, Sushma, Gautam AK 2019 - Two new additions to mycoflora (Hyphomycetes) of India. Studies in Fungi 4(1), 230-243, Doi 10.5943/sif/4/1/25

\begin{abstract}
Hyphomycetes are abundant and highly diverse in tropical areas such as India due to its climate and geographical landscape. However, several regions of the country remained unexplored or underexplored and many species remained undiscovered in the country. During mycological forays from the forests of Himachal Pradesh, two hyphomycetous fungi were collected. After detailed analysis of mycological characters like oval to oblong-ellipsoidal, muriform conidia with transverse, longitudinal, and oblique septa rounded or irregular at the ends and often with a protruding hilum, singly on short, unbranched conidiophores or sessile, in sporodochial conidiomata, it was found that these taxa belongs to the genus Berkleasmium. The further evaluation of morphological and microscopic characters revealed the identity of these fungi as $B$. pandani and B. typhae. Review of literature reveals that both are new records for India. Synoptic table of all the accepted species (both asexual morph and sexual morph) along with checklist of Berkleasmium from India is also provided.
\end{abstract}

Key words - Anamorphic fungi - biodiversity - taxonomy

\section{Introduction}

Himachal Pradesh is a state of Indian union situated between $30^{\circ} 22^{\prime} 40^{\prime \prime}$ to $33^{\circ} 12^{\prime} 40^{\prime \prime}$ along North latitudes and $75^{\circ} 45^{\prime} 55^{\prime \prime}$ to $79^{\circ} 04^{\prime} 20^{\prime \prime}$ along East longitudes. The altitude varies from 350 meters to 6975 meters above mean sea level. Terrestrial fungi play an important role in nutrient cycling and are important part of forest ecosystem of Himalayan region. Most of the studies of fungal diversity in the region are restricted mainly to Agaricomycetes (Prasher \& Ashok 2013, Ashok \& Prasher 2014a, b, Prasher 2015). There are sporadic reports of hyphomycetes primarily pertaining to the plant pathogenic fungi affecting the crops of this area (Bilgrami et al. 1991, Jamaluddin et al. 2004). India is an active centre of research of fungal biodiversity, with a high proportion of genera and species first described in this country (Subramanian 1971, Bilgrami et al. 1991, Jamaluddin et al. 2004). In spite of this intensive research, new species and records of hyphomycetes are still discovered in India (Kumar \& Singh 2018, Kumar et al. 2018, Rajeshkumar et al. 2018, Verma et al. 2019).

The genus Berkleasmium was established by Zobel in 1854 with B. cordaeanum Zobel as the type species. Hughes (1958) considered B. cordaeanum as a synonym of Sporidesmium concinnum Berkeley and proposed it as Berkleasmium concinnum (Berk.) S. Hughes. 


\section{Asexual morph}

There are two main morphological types have been reported by Lu et al. (2018)

1. Forming sporodochia, having short or flexuous conidiophores and producing muriform, ellipsoidal conidia broad-cylindrical, multicellular, with large and fairly regular cells, fuscous, borne on short conidiophores which become obscure with maturity (Moore 1958, Ellis 1971, Lu et al. 2018).

2. Effuse, helicosporous, gregarious, mycelium partly immersed, partly superficial, brown, septate, branched hyphae, with masses of crowded, glistering conidia; conidiophores erect, arising as lateral branches from creeping hyphae, macronematous, mononematous, 0-3-septate, brown, smoothwalled; conidiogenous cells holoblastic, mono- to polyblastic, integrated, sympodial, terminal, cylindrical, truncate at apex, brown, smooth-walled; conidia acrogenous, helicoid, tapering to apex and base, solitary, coiled 1-3 times, with elongated basal cell, rounded at tip, septate, slightly constricted at septa (Lu et al. 2018).

\section{Sexual Morph}

Ascomata superficial, seated on a subiculum, scattered, solitary, subglobose to globose, dark brown to black, with ostiole. Ascomata covered by setae. Setae multi-septate, thick-walled, brown to black, straight or slightly curved. Peridium composed of textura angularis. Hamathecium comprising, branched, filiform, septate, pseudoparaphyses. Asci bitunicate, cylindrical, pedicellate, apically with 8 ascospores. Ascospores biseriate, fusiform, hyaline, smooth-walled, tapering towards the ends, slightly curved, guttulate, multi-septate, not constricted at septa (Lu et al. 2017, 2018).

During the survey of saprophytic conidial fungi, two interesting hyphomycetes were collected. Morphological analysis confirmed that these taxa are Berkleasmium pandani McKenzie and B. typhae Somrithipol \& E.B.G. Jones were collected. This is the first report of both taxa from India (Bilgrami et al. 1991, Jamaluddin et al. 2004). Moreover, this communication is in continuation with our earlier reports on hyphomycetes (Prasher \& Verma 2014a, b, 2015a, b, c, 2016, Adamčik et al. 2015, Buyck et al. 2017, Verma et al. 2019).

\section{Materials \& Methods}

Decaying culms, twigs and dead wood and bark were collected and kept in paper bags. Then the specimens were brought to the laboratory. The specimens were mounted in $4 \% \mathrm{KOH}$, lactophenol and cotton blue $0.01 \%$ in lactophenol (Kirk et al. 2008), and were examined for microscopic characters using a Matrix stereo trinocular microscope (VL-Z60) and transmission microscope (VRS-2f) for macroscopic and microscopic characters. All measurements were taken with the help of Pro MED software. The scanning electron microscopic studies were conducted with a JSM-6100 (JEOL Ltd.) microscope. The material was vacuum-dried in an oven for 24 hours, mounted and sputtered with gold for 60 seconds, and photographed. The specimens were deposited in the herbarium of the Department of Botany, Panjab University, Chandigarh, India (PAN)

\section{Results}

Berkleasmium pandani McKenzie, Mycotaxon 104: 24 (2008)

Figs 1, 2

Index Fungorum: IF511285

Colonies on natural substratum forming black punctiform scattered, pulvinate, shiny or glistening, more or less circular sporodochia. Mycelium mainly immersed in the substratum. Stroma none. Setae and hyphopodia absent. Conidiophores reduced to conidiogenous cell, differentiated, single, aseptate, hyaline, thin-walled, smooth. Conidiogenous cell holoblastic, determinate, 12-20 × 8-19 $\mu \mathrm{m}$. Conidia solitary, dry, acrogenous, brown, smooth, oval to oblongellipsoidal, muriforrn with transverse, longitudinal, and oblique septa, sometimes slightly constricted at the median septa and other septa, 26-35 × 18-22.5 $\mu \mathrm{m}$. Conidiogenous cell usually remaining attached to base of conidium at maturity. 
Material examined - India, Himachal Pradesh, Near Kandaghat (Solan), on bark of Eucalyptus tereticornis, 5 April 2014, Rajnish Kumar Verma, PAN (32722).

Remarks - Our collection morphologically resembles with B. pandani (McKenzie 2008). The Himalayan collection differs from type in having smaller size of conidiogenous cell i. e 16-26 $\times 10$ 19 in type and 12-20 × 8-19 $\mu \mathrm{m}$ in Himalayan collection, but we considered it as same species. This is the first record of $B$. pandani from India.

Berkleasmium typhae Somrithipol \& E.B.G. Jones, Fungal diversity 12: 170 (2003). $\quad$ Figs 3, 4 Index Fungorum: IF372008

Colonies on natural substratum forming punctiform, scattered, brown, shiny sporodochia. Mycelium immersed in the substratum. Stroma none. Setae and hyphopodia absent. Conidiogenous cells terminal on the conidiophores, holoblastic 10-14 × 8-10 $\mu \mathrm{m}$. Conidia solitary, oval to ellipsoidal, muriform, constricted at the septa, often with a dark median septum, pale brown to brown, smooth, $20-29 \times 15.5-18.9 \mu \mathrm{m}$. Conidiogenous cell sometimes remaining attached to base of conidium at maturity.

Material examined - India, Himachal Pradesh, on the way Kuthera to Morshingi (Bilaspur), on bark of Eucalyptus species, 19 January 2013, Rajnish Kumar Verma, PAN (32723).

Remarks - Our new collection morphologically resembles B. typhae (Somrithipol \& Jones 2003). The species is the first report of B. typhae from India (Bilgrami et al. 1991, Jamaluddin et al. 2004).

\section{Discussion}

Presently there are 45 accepted species of Berkleasmium (Bussaban et al. 2001, Seifert et al. 2011, Whitton et al. 2012, Qu et al. 2013, Hüseyin et al. 2014, Tanney \& Miller 2017, Tibpromma et al. 2017, Lu et al. 2018). Among these species, B. aquaticum reported as sexual morph while two species (viz. B. fusiformae and $B$. thailandicum) are reported with both sexual and asexual morphs. Of the 44 asexual morphs four species namely B. guangxiense, B. latisporum, B. longisporum and $B$. thailandicum are having helicoid conidia rest have dictyoconidia. Table 1 and 2 summarizes synopsis of Berkleasmium.

Table 1 Synopsis of Berkleasmium species with asexual morph

\begin{tabular}{|c|c|c|c|c|}
\hline \multicolumn{5}{|c|}{ Species with Dictyoconidia } \\
\hline $\begin{array}{l}\text { Sr. } \\
\text { no. }\end{array}$ & Species & Conidiophores $(\mu \mathrm{m})$ & Conidia $(\mu \mathrm{m})$ & Reference \\
\hline 1. & B. ariense & $\begin{array}{l}\text { flexuous, unbranched, } \\
2-5\end{array}$ & $\begin{array}{l}119-238 \times 41-60 \text {, solitary, } \\
\text { dry or sticky, acrogenous, } \\
\text { simple, clavate, ellipsoidal, } \\
\text { oblong or irregular, } \\
\text { muriform, pale to dark } \\
\text { brown or blackish brown, } \\
\text { hilum not protruding; } \\
\text { conidial base } 11-15 \text {. Young } \\
\text { conidia hyaline or } \\
\text { subhyaline, } 56 \times 17\end{array}$ & $\begin{array}{l}\text { Tibpromma et } \\
\text { al. } 2017\end{array}$ \\
\hline 2. & B. atropicale & - & $\begin{array}{l}30-50 \times 21-38 \text { broadly } \\
\text { elliptical, sometimes ovoid } \\
\text { or obovoid }\end{array}$ & $\begin{array}{l}\text { Whitton et al. } \\
2012\end{array}$ \\
\hline 3. & B. atrovirens & $13-16 \times 1.5-3.5$ & $\begin{array}{l}\text { Irregularly muriform, 30-37 } \\
\times 15-17\end{array}$ & $\begin{array}{l}\text { Zhao \& Zhang } \\
2004\end{array}$ \\
\hline 4. & B. concinnum & $30 \times 2-5$ & 60-124 × 24-31, muriform & Hughes 1958 \\
\hline 5. & B. conglobatum & - & $36.5-99 \times 26-47$ & Moore 1958 \\
\hline
\end{tabular}


Table 1 Continued.

\begin{tabular}{|c|c|c|c|c|}
\hline $\begin{array}{l}\text { Sr. } \\
\text { no. }\end{array}$ & Species & Conidiophores $(\mu \mathrm{m})$ & Conidia $(\mu \mathrm{m})$ & Reference \\
\hline 6. & B. correae & $2-5 \times 2-2.5$ & $\begin{array}{l}26-44 \times 16-24 \text {, broadly } \\
\text { ellipsoidal }\end{array}$ & Yip 1988 \\
\hline 7. & B. corticola & - & $\begin{array}{l}26.5-34 \times 18.5-26 \text {, } \\
\text { multicellular, with } 1 \text { or } 2 \\
\text { subtending cells }\end{array}$ & Moore 1959 \\
\hline 8. & B. crunisia & $45-47.5 \times 10$ & $\begin{array}{l}\text { muriform, oval to } \\
\text { ellipsoidal, pale brown, } \\
\text { smooth, 35-100 × 17.5-30 }\end{array}$ & $\begin{array}{l}\text { Pinnoi et al. } \\
2007\end{array}$ \\
\hline 9. & B. daphniphylli & - & $16.5-28 \times 10-13$ & $\begin{array}{l}\text { Zhang et al. } \\
2009\end{array}$ \\
\hline 10. & B. dudkae & $\begin{array}{l}\text { up to } 25 \text { long, } 1.8- \\
2.3(-2.9) \text { wide }\end{array}$ & $\begin{array}{l}\text { oval to obovoid, smooth, } \\
\text { regularly muriform, }(13-) \\
14-17(-17.5) \times 12.5-15\end{array}$ & $\begin{array}{l}\text { Hüseyin et al. } \\
2014\end{array}$ \\
\hline 11. & B. fusiforme & up to 8 long, 3-5 wide & $\begin{array}{l}\text { fusiform, oval to ellipsoidal, } \\
18-29 \times 10-14\end{array}$ & Qu et al. 2013 \\
\hline 12. & B. granulosum & - & $\begin{array}{l}\text { 29-66 × 16.5-18.5, profile } \\
\text { irregular, multicellular }\end{array}$ & Moore 1958 \\
\hline 13. & B. inflatum & $\begin{array}{l}\text { 39-80 long, 3-4 wide } \\
\text { at base, 7-19 apex }\end{array}$ & ellipsoidal, 31.5-49 × 16-26 & $\begin{array}{l}\text { Holubová- } \\
\text { Jechová } 1987\end{array}$ \\
\hline 14. & B. juglandis & $7-15 \times 8-13$ & $20-25 \times 14-18$ & Qu et al. 2013 \\
\hline 15. & B. leonense & $\begin{array}{l}\text { Up to } 25 \text { long, } 1-3 \\
\text { wide }\end{array}$ & $\begin{array}{l}20-27 \times 15-17 \times 6-8, \\
\text { muriform, broadly elliptical, } \\
\text { flattened, smooth }\end{array}$ & Ellis 1976 \\
\hline 16. & B. lingual & - & $\begin{array}{l}65-89.5 \times 18.5-24 \\
\text { multicellular, without } \\
\text { subtending cells, cylindrical } \\
\text { to slightly obclavate, } \\
\text { sometimes somewhat } \\
\text { curved, fuscous }\end{array}$ & Moore 1959 \\
\hline 17. & $\begin{array}{l}\text { B. } \\
\text { micronesicum }\end{array}$ & - & $\begin{array}{l}\text { 30-40 × 13-16, muriform, } \\
\text { cylindrical }\end{array}$ & $\begin{array}{l}\text { Matsushima } \\
1981\end{array}$ \\
\hline 18. & $\begin{array}{l}\text { B. } \\
\text { minutissimum }\end{array}$ & Absent & $\begin{array}{l}11.5-17 \times 8.5-11.5, \\
\text { subglobose to squarish- } \\
\text { subglobose to oval ellipsoid }\end{array}$ & Moore 1959 \\
\hline 19. & $\begin{array}{l}\text { B. } \\
\text { monilicellulare }\end{array}$ & $13-23 \times 4-5.2$ & $\begin{array}{l}27-39 \times 20-29 \text {, broadly } \\
\text { elliptical, pyriform or } \\
\text { sometimes almost spherical. }\end{array}$ & $\begin{array}{l}\text { Whitton et al. } \\
2012\end{array}$ \\
\hline 20. & B. moriforme & - & $\begin{array}{l}23.5-26.5 \times 18.5-22, \\
\text { muriform, pale brown } \\
\text { subtending cells either } \\
\text { numerous, tending to be } \\
\text { amorphous }\end{array}$ & Moore 1959 \\
\hline 21. & B. nigroapicale & $\begin{array}{l}\text { Up to } 22 \text { high, inflated } \\
\text { to } 6.5-10 \text { at the apex }\end{array}$ & $\begin{array}{l}24-27 \times 12.5-15 \text {, broadly } \\
\text { clavate, muriform }\end{array}$ & $\begin{array}{l}\text { Bussaban et al. } \\
2001\end{array}$ \\
\hline 22. & B. obovoides & 17 long, 6 wide & $\begin{array}{l}\text { ellipsoidal to obovoid, } \\
\text { regularly muriform, } 26-34 \times \\
\text { 19-24 }\end{array}$ & Qu et al. 2013 \\
\hline
\end{tabular}


Table 1 Continued.

\begin{tabular}{|c|c|c|c|c|}
\hline $\begin{array}{l}\text { Sr. } \\
\text { no. }\end{array}$ & Species & Conidiophores ( $\mu \mathrm{m})$ & Conidia $(\mu \mathrm{m})$ & Reference \\
\hline 23. & B. opacum & Absent & $\begin{array}{l}52-83 \times 31-41.5 \text {, verrucose, } \\
\text { subglobose to oval- } \\
\text { ellipsoid, }\end{array}$ & Moore 1959 \\
\hline 24. & B. osmaniae & $7.2-7.6 \times 3.6-7.2$ & $\begin{array}{l}18-75 \times 3.6-5.4 \text { at base, } \\
7.2-10.8 \text { at apex, oval- } \\
\text { clavate to cylindrical, } \\
\text { curved or straight, Smooth }\end{array}$ & $\begin{array}{l}\text { Rao \& Rao } \\
1964\end{array}$ \\
\hline 25. & B. pandani & $\begin{array}{l}\text { Reduced to } \\
\text { conidiogenous cell }\end{array}$ & $27-34 \times 18-22.5$ & McKenzie 2008 \\
\hline 26. & B. parmelielfae & $6-9 \times 4-5$ & $\begin{array}{l}40-55 \times 22-27, \text { muriform, } \\
\text { clavate to ellipsoidal, } \\
\text { rounded at the ends }\end{array}$ & $\begin{array}{l}\text { Rao \& Rao } \\
1964\end{array}$ \\
\hline 27. & B. parmeliellae & - & $40-45 \times 22-27$ & $\begin{array}{l}\text { Etayo \& } \\
\text { Diederich } 1995\end{array}$ \\
\hline 28. & $\begin{array}{l}\text { B. } \\
\text { phyllostachydis }\end{array}$ & - & $\begin{array}{l}\text { 17-25 × 9-13, muriform, } \\
\text { ellipsiodal to broadly } \\
\text { clavate }\end{array}$ & $\begin{array}{l}\text { Matsushima } \\
1983\end{array}$ \\
\hline 29. & B. pulcharum & $\begin{array}{l}\text { up to } 25 \text { long, } 2-5 \\
\text { thick }\end{array}$ & $\begin{array}{l}\text { muriform, brown, smooth, } \\
31-41(-46) \times 21-28(-32) \times \\
13-16 \text {, with }(3-) 4-5(-7) \\
\text { transverse and } 2 \\
\text { longitudinal thick, dark } \\
\text { septa, basal cell conical, } \\
\text { hyaline to sub-hyaline, } 3-5 \\
\times 2.5-5 \text {. }\end{array}$ & $\begin{array}{l}\text { Holubová- } \\
\text { Jechová \& } \\
\text { Mercado } \\
\text { Sierra1984 }\end{array}$ \\
\hline 30. & B. sansevieriae & $5-13 \times 2.6$ & $\begin{array}{l}\text { 15.5-26 × I0.5-15.5, } \\
\text { oblong, muriform }\end{array}$ & $\begin{array}{l}\text { Batista et al. } \\
1962\end{array}$ \\
\hline 31. & B. sinense & - & $\begin{array}{l}\text { broadly ellipsoidal to } \\
\text { obovoid, brown, paler } \\
\text { towards base, } \\
40-52 \times 20-32\end{array}$ & $\begin{array}{l}\text { Taylor \& Hyde } \\
2003\end{array}$ \\
\hline 32. & $\begin{array}{l}\text { B. } \\
\text { sutheppuiense }\end{array}$ & $15-17.5 \times 6-6.5$ & $\begin{array}{l}35-37 \times 22.5-25, \text { muriform, } \\
\text { subglobose, }\end{array}$ & $\begin{array}{l}\text { Bussaban et al. } \\
2001\end{array}$ \\
\hline 33. & B. taishanense & $\begin{array}{l}\text { Up to } 10 \text { long } \\
\text { (including } \\
\text { conidiogenous cell) } \\
\text { 1.5-2 wide with } \\
\text { bladder like swelling }\end{array}$ & $\begin{array}{l}\text { Ellipsoidal, clavate, ovovate } \\
\text { or pyriform, } 22-35 \times 15- \\
18.75\end{array}$ & $\begin{array}{l}\text { Zhao \& Zhang } \\
2004\end{array}$ \\
\hline 34. & B. talaumae & Absent & 25-51 × 19-28, oblong, & $\begin{array}{l}\text { Batista et al. } \\
1964\end{array}$ \\
\hline 35. & B. tetraploides & up to 15 long, $3-5$ wide & $\begin{array}{l}\text { pyriform, smooth, } 20-30 \times \\
12-19\end{array}$ & Qu et al. 2013 \\
\hline 36. & B. triglochinis & 4-6 long & $\begin{array}{l}10-18 \times 8-14 \text {, tuberculate } \\
\text { to more or less scabrid, } \\
\text { broadly oval to spherical or } \\
\text { pyriform, }\end{array}$ & Moore 1959 \\
\hline 37. & B. tropicale & $13-16 \times 3-4$ & $\begin{array}{l}\text { 50-57 × 28-34, muriform, } \\
\text { flattened, oval }\end{array}$ & Morris 1972 \\
\hline
\end{tabular}


Table 1 Continued.

\begin{tabular}{|c|c|c|c|c|}
\hline $\begin{array}{l}\text { Sr. } \\
\text { no. }\end{array}$ & Species & Conidiophores $(\mu \mathrm{m})$ & Conidia $(\mu \mathrm{m})$ & Reference \\
\hline 38. & B. typhae & - & $23-28.5 \times 15-19.5$ & $\begin{array}{l}\text { Somrithipol \& } \\
\text { Jones } 2003\end{array}$ \\
\hline 39. & B. zhejiangense & - & $\begin{array}{l}\text { oval to ellipsoidal, } 38-76 \times \\
19-33\end{array}$ & $\begin{array}{l}\text { Wongsawas et } \\
\text { al. } 2009\end{array}$ \\
\hline \multicolumn{5}{|c|}{ Species with Helicoid conidia } \\
\hline 1. & B. guangxiense & $\begin{array}{l}19-37 \times 3.5-5 \text {, pale } \\
\text { brown, } \\
\text { macronematous, erect, } \\
\text { short, cylindrical, } \\
\text { septate, smooth-walled }\end{array}$ & $\begin{array}{l}90-130(-144) \text { diam. and } \\
\text { conidial filament } 8-10 \text { wide, } \\
405-725(-800) \text { long, coiled } \\
21 / 2-31 / 2 \text { times when tightly } \\
\text { coiled, basal cell elongated, } \\
\text { rounded at tip, multiseptate, } \\
\text { up to } 62-\text { septate }\end{array}$ & $\begin{array}{l}\text { Lu et. al. 2017, } \\
2018\end{array}$ \\
\hline 2. & B. latisporum & - & $\begin{array}{l}\text { 95-120 diam. and conidial } \\
\text { filament } 12-14.5 \text { wide, } 540- \\
760 \text { long, coiled } 1 \frac{1}{2}-31 / 2 \\
\text { times when tightly coiled, } \\
\text { becoming loosely coiled in } \\
\text { the water, basal cell } \\
\text { elongated, rounded at the } \\
\text { tip, multi-septate, up to } 60 \text { - } \\
\text { septate }\end{array}$ & $\begin{array}{l}\text { Lu et al. 2017, } \\
2018\end{array}$ \\
\hline 3. & B. longisporum & $\begin{array}{l}\text { 0-3-septate, } 15-40 \times \\
\text { 5-7, brown, smooth- } \\
\text { walled. }\end{array}$ & $\begin{array}{l}\text { solitary, acrogenous, } \\
\text { helicoid, basal cell } \\
\text { elongated, rounded at tip, } \\
110-180 \text { diam. and conidial } \\
\text { filament } 11-15 \text { wide in the } \\
\text { broadest, tapering to } 4.5-5.5 \\
\text { lm wide near apex and base, } \\
\text { 1190-1540 long, 100-122 } \\
\text { septate, slightly constricted } \\
\text { at septa, coiled } 2-3 \text { times, } \\
\text { becoming loosely coiled or } \\
\text { uncoiled in water, brown, } \\
\text { smooth-walled }\end{array}$ & Lu et al. 2018 \\
\hline 4. & B. thailandicum & $\begin{array}{l}10-24 \times 4-6 \text {, pale } \\
\text { brown, } \\
\text { macronematous, erect, } \\
\text { short, cylindrical, } \\
\text { septate }\end{array}$ & $\begin{array}{l}\text { 89-155 diam. and conidial } \\
\text { filament 10.5-14 (-15) } \\
\text { wide, 630-950 long, coiled } \\
2-3 \text { times when tightly } \\
\text { coiled, up to 60-septate }\end{array}$ & Lu et al. 2017 \\
\hline
\end{tabular}


Table 2 Synopsis of Berkleasmium species with sexual morph

\begin{tabular}{|c|c|c|c|c|c|c|c|}
\hline $\begin{array}{l}\text { Sr. } \\
\text { no. }\end{array}$ & Species & Ascomata $(\mu \mathrm{m})$ & $\begin{array}{l}\text { Setae } \\
(\mu \mathrm{m})\end{array}$ & $\begin{array}{l}\text { Peridium } \\
(\mu \mathrm{m})\end{array}$ & $\begin{array}{l}\text { Asci } \\
(\mu \mathrm{m})\end{array}$ & $\begin{array}{l}\text { Ascospores } \\
(\mu \mathrm{m})\end{array}$ & Reference \\
\hline
\end{tabular}



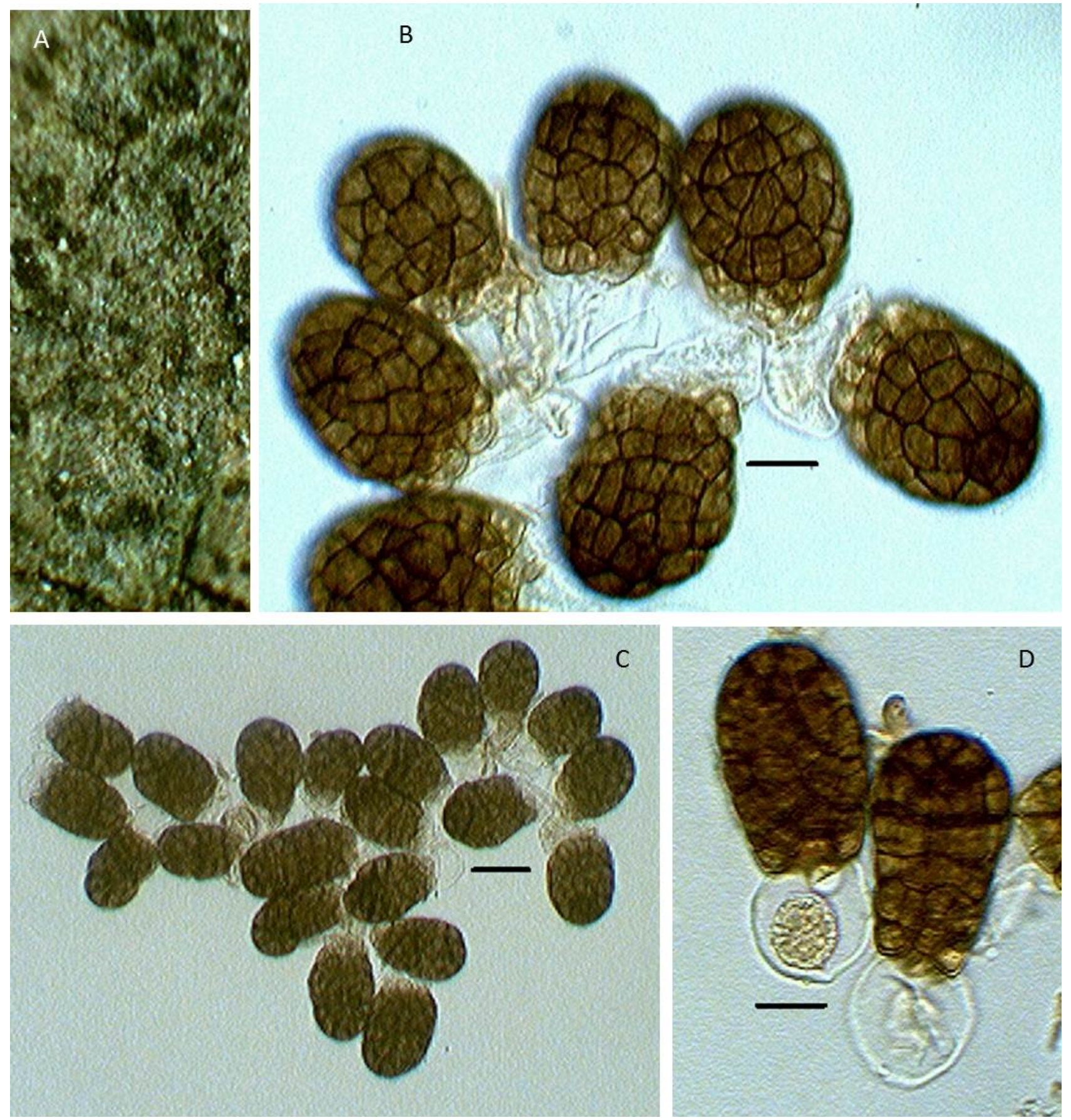

Fig. 1 - Berkleasmium pandani A Colonies on natural substratum. B, C Squashed mount of sporodochium. D Conidia with conidiogenous cell. Scale bars: A, $D=10 \mu \mathrm{m}, \mathrm{C}=20 \mu \mathrm{m}$. 


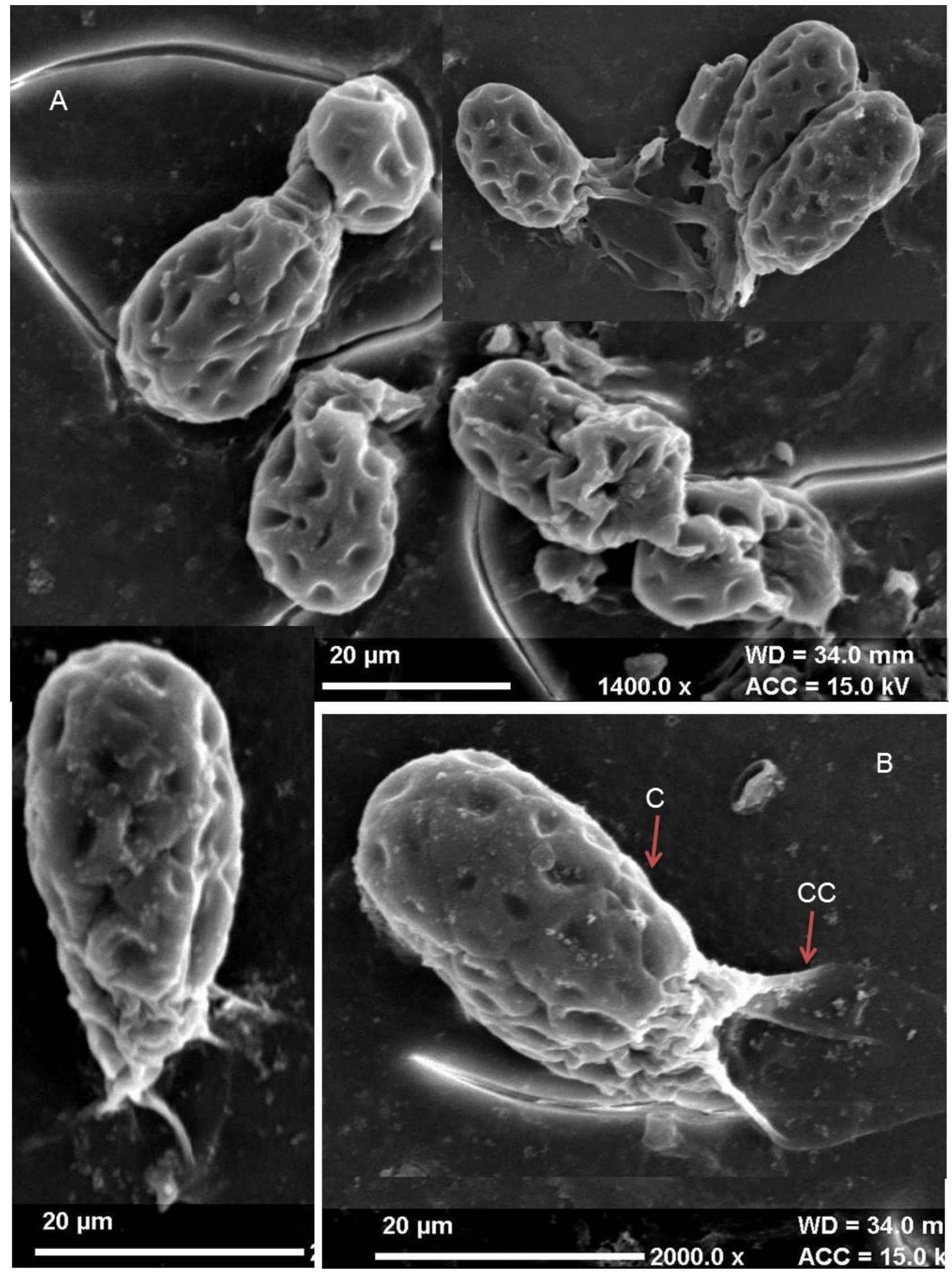

Fig. 2 - Berkleasmium pandani (Scanning electron micrograph) A, B Conidia. (CC= Conidiogenous cell, $C=$ Conidium). Scale bar: A, B $=20 \mu \mathrm{m}$. 

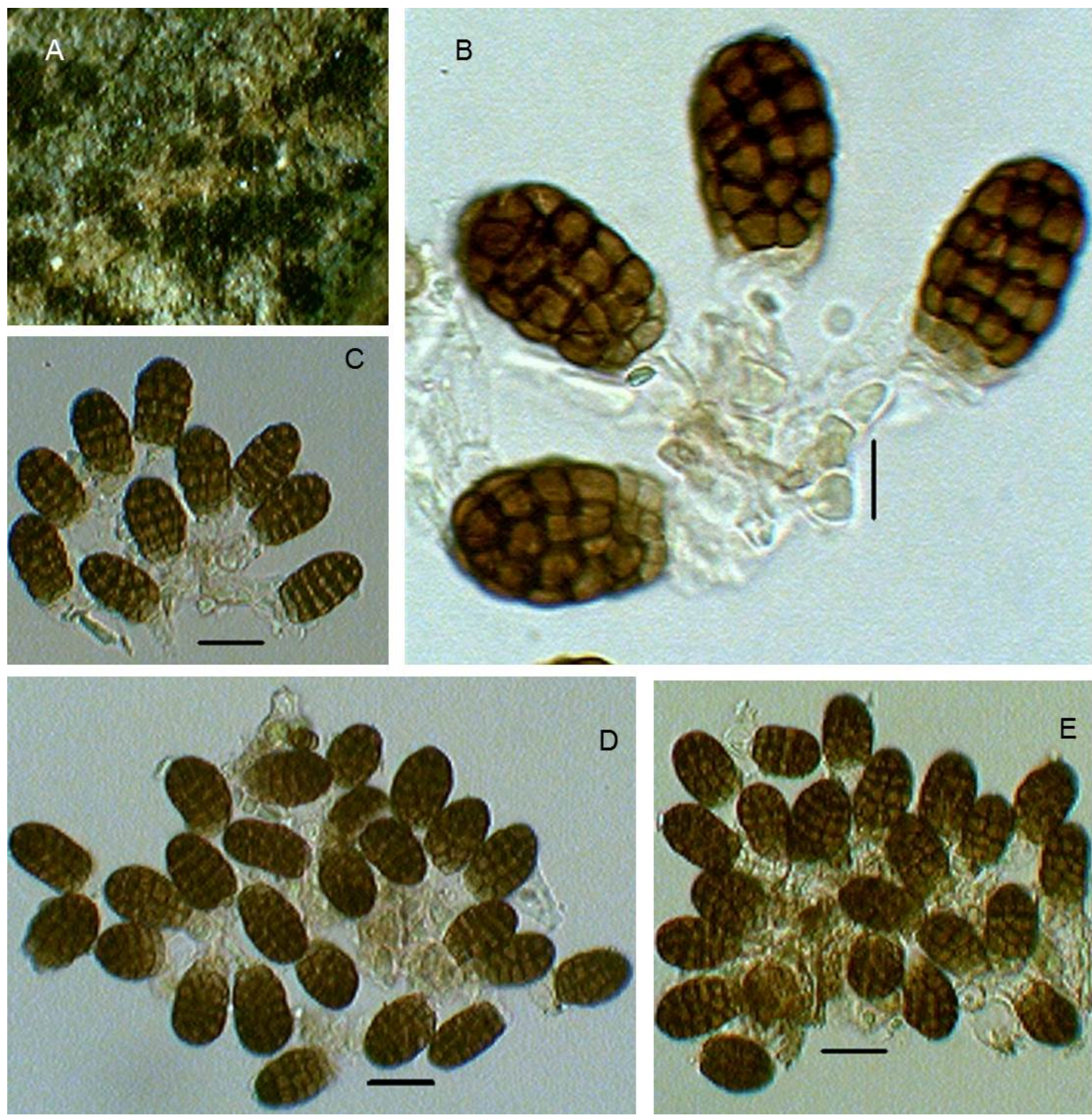

Fig. 3 - Berkleasmium typhae A Colonies on natural substratum. B-E Squashed mount of Sporodochium. Scale bars: $B=10 \mu \mathrm{m}, \mathrm{C}-\mathrm{E}=20 \mu \mathrm{m}$. 

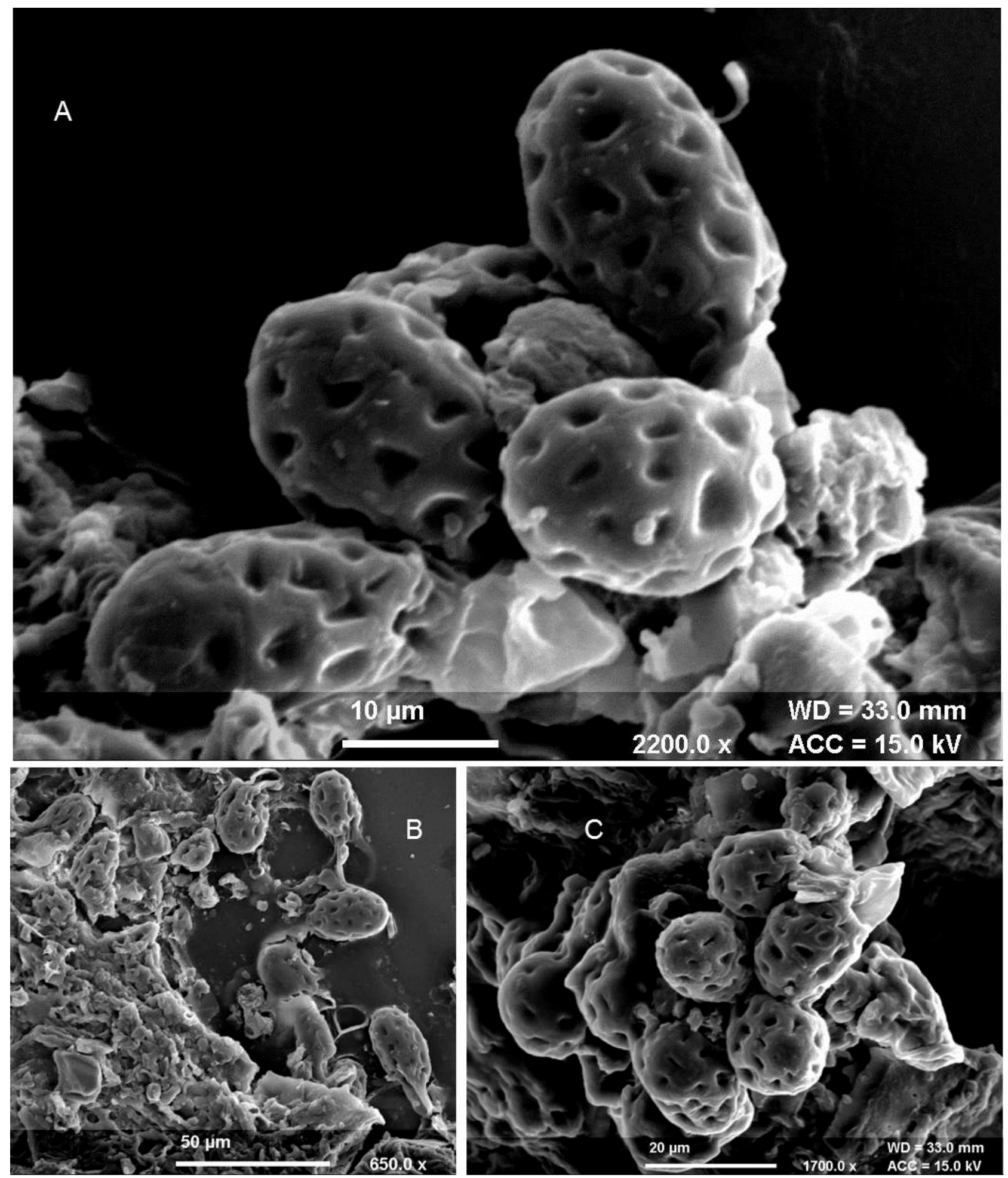

Fig. 4 - Berkleasmium typhae (Scanning electron micrograph) A, C Part of sporodochium showing conidia. B Conidia. Scale bars: $A=10 \mu \mathrm{m}, \mathrm{B}=50 \mu \mathrm{m}, \mathrm{C}=20 \mu \mathrm{m}$

\section{Checklist of Berkleasmium from India.}

Berkleasmium ariense Rajeshk. \& Marathe, in Tibpromma et al., Fungal Diversity 83: 35 (2017). Distribution: Maharashtra, Tamhini Village, on unidentified stem litter (Tibpromma et al. 2017) Berkleasmium conglobatum (Cooke \& Ellis) R.T. Moore, Mycologia 50(5): 687 (1959) [1958] 三Sporidesmium conglobatum Cooke \& Ellis, Grevillea 8(no. 45): 11 (1879)

Distribution: On bark of Syzygium fruiticosum New Delhi (Chowdhry 1974)

Berkleasmium corticola (P. Karst.) R.T. Moore, Mycologia 51(5): 735 (1961) [1959] 
$\equiv$ Sporidesmium corticola (P. Karst.) Mussat [as ‘corticolum'], in Saccardo, Syll. fung. (Abellini) 15: 398 (1901)

三Sporidesmium moriforme subsp. corticola P. Karst., Meddn Soc. Fauna Flora fenn. 14: 99 (1887)

Distribution: On dead bark of Tectona grandis, Jabalpur Madhya Pradesh (Sharma 1980), on Syzygium fruiticosum Agra Uttar Pradesh (Sharma et al. 1976)

Berkleasmium leonense M.B. Ellis, More Dematiaceous Hyphomycetes (Kew): 69 (1976)

Dead twigs of Odina wodier, Moonar, kerala (Rao \& Varghese 1979, Varghese \& Rao 1979);

Distribution: unidentified dead wood, Dehradun, Uttrakhand (Begum et al. 1978);

Berkleasmium osmaniae P. Rag. Rao \& D. Rao, Mycopath. Mycol. appl. 22: 314 (1964)

Distribution: Dead culms of gramineae, hydrabad Andhra Pradesh (Rao \& Rao 1964)

\section{Acknowledgements}

The authors are thankful to Ministry of Environment and Forests, Government of India for the financial assistance (vide letter no. 14/26/2008-ERS/RE dt. 06.06.2010), UGC (SAP, DRS III) and Chairperson Department of Botany Panjab University Chandigarh for providing infrastructural and laboratory facilities.

\section{References}

Adamčik S, Cai L, Chakraborty D, Chens XH et al. 2015 - Fungal Biodiversity Profiles 1-10. Cryptogamie Mycologie 36 (2), 121-166. Doi: http://dx.doi/10.7872/crym/v36.iss2.2015.1

Ashok D, Prasher IB. 2014a - Wood rotting non-gilled Agaricomycetes new to India. Journal on new biological reports. 3(1), 04-08.

Ashok D, Prasher IB. 2014b - Some interesting wood rotting Agaricomycetes: New to India. Journal on new biological reports. 3(2), 155-158.

Batista JL, Bezerra, Cavalcanti. 1962 - Publicaçoes do Instituto de Micologia da Universidade do Recife 342: 8

Batista AC, Cavalcanti W de A. 1964 - Trichobolbus n. gen. e outros deuteromycetes curiosos. Rivista di Patologia Vegetale. 4, 559-567

Begum R, Manoharachary C, Rizwana AR. 1978 - Additions to the fungi of india. Acta botanica indica 6, 190-191

Bilgrami KS, Jamaluddin, Rizwi MA. 1991 - Fungi of India List and References. Today and tomorrow's Printers \& Publishers, New Delhi, India

Bussaban B, Lumyong S, Lumyong P, McKenzie EHC, Hyde KD. 2001 - A synopsis of the genus Berkleasmium with two new species and new records of Canalisporium caribense from Zingiberaceae in Thailand. Fungal Diversity 8, 73-85.

Buyck B, Duhem B, Das K, Yawardena RSJ et al. 2017 - Fungal Biodiversity Profiles 21-30. Cryptogamie Mycologie 38 (1), 101-146.

Chowdhry PN. 1974- Two fungi from jamun tree. Indian phytopathology. 27, 423-425

Ellis MB. 1971 - Dematiaceous Hyphomycetes. Commonwealth Mycological Instititute, Kew, UK.

Ellis MB. 1976 - More Dematiaceous Hyphomycetes. Commonwealth Mycological Instititute, Kew, UK.

Etayo J, Diederich P. 1995 - Lichenicolous fungi. from the western Pyrenees, France and Spain. I, 205-221.

Holubová-Jechová V, Mercado Sierra A. 1984 - Studies on Hyphomycetes from Cuba II. Hyphomycetes from the Isla de la Juventud. Ceská Mykologie 38(2), 96-120.

Holubová-Jechová V. 1987 - Studies on hyphomycetes from Cuba V. Six new species of dematiaceous hyphomycetes from Havana Province. Ceská Mykologie 41(1), 29-36.

Hughes SJ. 1958 - Revisiones Hyphomycetum aliquot cum appendice de nominibus rejiciendis. Canadian Journal of Botany. 36(6), 727-836.

Hüseyin E, Selçuk F, Churakov BP. 2014 - A new species of Berkleasmium from Ulyanovsk, Russia. Mycosphere 5(3), 462-466. Doi: 10.5943/mycosphere/5/3/8. 
Jamaluddin, Goswami MG, Ojha BM. 2004 - Fungi of India 1989-2001. Scientific Publishers, Jodhpur, India.

Kirk PM, Cannon PF, Minter DW, Stalpers JA. 2008 - Dictionary of the Fungi. $10^{\text {th }}$ edn. CAB International, Wallingford, UK.

Kumar S, Singh R, Singh DP, Kamal. 2018 - Crousobrauniella, an interesting new foliicolous hyphomycetous genus from Uttar Pradesh, India. Kavaka, 50, 64-68.

Kumar S, Singh R. 2018 - Curvularia martyniicola, a new species of foliicolous hyphomycetes on Martynia annua from India. Studies in Fungi 3(1), 27-33, Doi: 10.5943/sif/3/1/4

Lu YZ, Boonmee S, Liu JK, Hyde KD et al. 2017 - Novel Neoacanthostigma Species from Aquatic Habitats. Cryptogamie, Mycologie 38(2), 169-190.

Doi: doi.org/10.7872/crym/v38.iss2.2017.169

Lu YZ, Liu JK, Hyde KD, Jeewon R et al. 2018 - A taxonomic reassessment of Tubeufiales based on multi-locus phylogeny and morphology. Fungal Diversity, 92, 131-344.

Matsushima T. 1981 - Matsushima Mycological Memoirs 2. Matsushima Mycological Memoirs. 2, $1-68$.

Matsushima T. 1983 - Matsushima Mycological Memoirs 3. Matsushima Mycological Memoirs. 3:1-90.

McKenzie EHC. 2008 - Two new dictyosporous hyphomycetes on Pandanaceae. Mycotaxon. 104, 23-28

Moore RT. 1958 - Deuteromycetes I: The Sporidesmium complex. Mycologia. 50, 681-692.

Moore RT. 1959 - The genus Berkleasmium. Mycologia. 51(5), 734-739.

Morris EF. 1972 - Costa Rican Hyphomycetes. Mycologia. 64:887-896

Pinnoi A, Jeewon R, Sakayaroj J, Hyde KD, Jones EBG. 2007 - Berkleasmium crunisia sp. nov. and its phylogenetic affinities to the Pleosporales based on 18S and 28S rDNA sequence analyses. Mycologia. 99(3), 378-384.

Prasher IB, Ashok D. 2013 - A Checklist of wood-rotting fungi (non-gilled Agaricomycotina) of Himachal Pradesh. Journal on new biological reports. 2(2), 71-98

Prasher IB, Verma RK. 2014a - Taeniolina echinata- A new species of hyphomycetes (anamorphic) fungus from North India. Kavaka. 43, 11- 13

Prasher IB, Verma RK. 2014b - Four interesting Hyphomycetes from Himachal Pradesh. Journal on New Biological Reports. 3(3), 159 - 166.

Prasher IB. 2015 - Wood-rotting non-gilled Agaricomycetes of Himalayas. Fungal Diversity Research Series. 1-653

Prasher IB, Verma RK. 2015a - Two new species of Dictyosporium from India. Phytotaxa. 204 (3), 193-202. http://dx.doi.org/10.11646/phytotaxa.204.3.2

Prasher IB, Verma RK. 2015b - Some new and interesting Hyphomycetes from North Western Himalayas, India. Nova Hedwigia, 100(1-2), 269-277.

Doi: http://dx.doi.org/10.1127/nova_hedwigia/2014/2015

Prasher IB, Verma RK. 2015c - Neosporidesmium appendiculatus sp. nov. from North- Western India. Mycological Progress. 14: 87. Doi: http://dx.doi.org/10.1007/s11557-015-1112-5

Prasher IB, Verma RK. 2016 - The genus Monodictys from Himachal Pradesh. Kavaka. 47, 138142.

Qu C, Yin G, Zhao G, Cui B, Liu X. 2013 - Three new species of Berkleasmium (Hyphomycetes) from China. Nova Hedwigia, 98 (1-2), 151-161.

Rajeshkumar KC, Bhat DJ, Lad SS, Wijayawardene NW et al. 2018 - Morphology and phylogeny of Tamhinispora srinivasanii sp. nov. (Tubeufiaceae) from northern Western Ghats, India. Phytotaxa 346 (1), 113-120.

Rao PR, Rao D. 1964 - Berkleasmium Zobel from India. Mycopathologia et Mycologia Applicata. 22(4), 311-314.

Rao VG, Varghese MKI. 1979 - Fungi imperfecti from kerala, S. India-1. Biovigyanam. 5, 77-80

Seifert K, Morgan-Jones G, Gams W, Kendrick BW. 2011 - The Genera of Hyphomycetes. CBS Biodiversity Series vol. 9. 
Sharma KD, Agarwal DK, Choudhury PN. 1976 - Two dictyosporus dematiaceous hyphomycetes from Syzygium. Indian journal of microbiology. 16:43.

Sharma ND. 1980 - Some additions to fungi of India VIII. Journal of Indian Botanical society. 59, 72-79.

Somrithipol S, Jones EBG. 2003 - Berkleasmium typhae sp. nov., a new hyphomycete on narrowleaved cattail (Typha angustifolia) from Thailand. Fungal Diversity. 12, 169-172.

Subramanian CV. 1971 - Hyphomycetes: an account of Indian species, except Cercosporae. 1-930

Tanney J, Miller AN. 2017 - Asexual-sexual morph connection in the type species of Berkleasmium. IMA Fungus. 8, 99-105.

Taylor JE, Hyde KD. 2003 - Microfungi of tropical and temperate palms. 12, 1-459.

Tibpromma S, Hyde KD, Jeewon R, Maharachchikumbura SSN et al. 2017 - Fungal diversity notes 491-602: taxonomic and phylogenetic contributions to fungal taxa. Fungal Diversity. 83, 1261.

Varghese MKI, Rao VG. 1979 - Some additions to the Indian Fungi. J. Univ. Poona. 52, 309-312.

Verma RK, Prasher IB, Sushma. 2019 - New records of hyphomycetous fungi from North Western Himalayas. Journal on New Biological Report 8(1), 1-7.

Whitton SR, Mckenzie EHC, Hyde KD. 2012 - Fungi associated with Pandanaceae. Fungal Diversity Research Series. 21, 1-458.

Wongsawas M, Wang HK, Hyde KD, Lin FC. 2009 - Two new hyphomycetes from submerged wood collected in China. Sydowia 61 (2), 345-351.

Yip HY. 1988 - Berkleasmium Correae sp. nov. on leaf hairs of Correa lawrenciana. Australasian Plant Pathology. 17(2), 31-33.

Zhang K, Ma J, Ma LG, Zhang XG. 2009 - A new species of Berkleasmium from Chongqing, China. Mycotaxon. 108, 5-7.

Zhao GZ, Zhang TY. 2004 - Notes on dictyosporic hyphomycetes from China IV. The genus Berkleasmium. Mycotaxon. 89(2), 241-244.

Zobel. 1854 - Icones Fungorum 6:4 